\title{
TYPE DECOMPOSITION OF A PSEUDOEFFECT ALGEBRA
}

\author{
DAVID J. FOULIS, SYLVIA PULMANNOVÁ ${ }^{\otimes}$ and ELENA VINCEKOVÁ
}

(Received 16 January 2010; accepted 4 December 2010)

Communicated by G. A. Willis

\begin{abstract}
Effect algebras, which generalize the lattice of projections in a von Neumann algebra, serve as a basis for the study of unsharp observables in quantum mechanics. The direct decomposition of a von Neumann algebra into types I, II, and III is reflected by a corresponding decomposition of its lattice of projections, and vice versa. More generally, in a centrally orthocomplete effect algebra, the so-called type-determining sets induce direct decompositions into various types. In this paper, we extend the theory of type decomposition to a (possibly) noncommutative version of an effect algebra called a pseudoeffect algebra. It has been argued that pseudoeffect algebras constitute a natural structure for the study of noncommuting unsharp or fuzzy observables. We develop the basic theory of centrally orthocomplete pseudoeffect algebras, generalize the notion of a type-determining set to pseudoeffect algebras, and show how typedetermining sets induce direct decompositions of centrally orthocomplete pseudoeffect algebras.
\end{abstract}

2010 Mathematics subject classification: primary 06C15; secondary 17C65, 46L45.

Keywords and phrases: (pseudo)effect algebra, (pseudo)MV algebra, orthomodular poset, orthomodular lattice, boolean algebra, von Neumann algebra, JW algebra, Loomis dimension lattice, types I, II, and III.

\section{Introduction}

The classic theorem that a von Neumann algebra decomposes uniquely as a direct sum of subalgebras of types I, II, and III, [4, I, Section 8], [23] has played a prominent role both in the development of the theory of von Neumann algebras and in the applications of this theory in mathematical physics. Analogous type-decomposition theorems were featured in subsequent work on various generalizations of von Neumann algebras, including studies of AW* algebras [19], Baer*-rings [20], and JW algebras [27]. For a von Neumann algebra $A$, and for the aforementioned generalizations, the subset $P$ of all projections (self-adjoint idempotents) in $A$ forms an orthomodular lattice $[1,17]$,

The second and third authors were supported by the Research and Development Support Agency under contract no. LPP-0199-07; grant VEGA 2/0032/09, Center of Excellence SAS-Quantum Technologies; and ERDF OP R \& D Project CE QUTE ITMS 26240120009 and meta-QUTE ITMS 26240120022.

(C) 2011 Australian Mathematical Publishing Association Inc. 1446-7887/2011 \$16.00 
and the decomposition of $A$ into types induces a corresponding direct decomposition of the orthomodular lattice $P$. Conversely, a direct decomposition of $P$ yields a directsum decomposition of the enveloping algebra $A$. These connections between directsum decompositions of $A$ and direct decompositions of $P$ have motivated a number of studies of direct decompositions of more general orthomodular lattices.

The type-decomposition theorem for a von Neumann algebra is dependent on von Neumann-Murray dimension theory; likewise, the early type-decomposition theorems for orthomodular lattices were corollaries of the lattice-based dimension theories of Loomis [21] and Maeda [22]. The work of Loomis and Maeda was further developed by Ramsay [25] who proved that an arbitrary complete orthomodular lattice is uniquely decomposed into four special direct summands, one of which can be organized into a Loomis dimension lattice. More recent and considerably more general results on type decomposition based on dimension theory can be found in the monograph of Goodearl and Wehrung [15].

In [18, Section 7] Kalmbach obtained decompositions of an arbitrary complete orthomodular lattice into direct summands with various special properties, without employing lattice dimension theory per se. Moreover, Ramsay's fourfold decomposition is a special case of Kalmbach's theory. In [2], Carrega et al. obtained the direct decompositions of Kalmbach and Ramsay by methods more in the spirit of universal algebra.

In [13], the decomposition theory of Kalmbach, Carrega, and others was extended to the class of centrally orthocomplete effect algebras by employing the notion of a typedetermining set. Effect algebras [6, 10] are very general partially ordered algebraic structures, originally formulated as an algebraic base for the theory of measurement in quantum mechanics. Special cases of lattice-ordered effect algebras are orthomodular lattices and the MV algebras of Chang [3].

The notion of a (possibly) noncommutative effect algebra, called a pseudoeffect algebra, was introduced and studied in a series of papers by Dvurečenskij and Vetterlein [5, 7, 8]. See [9] for an indication of the utility of pseudoeffect algebras, not only in quantum measurement theory, but also in the study of the human brain, big computer networks, economic systems, and even in situations met in everyday life.

Whereas a prototypical example of an effect algebra is the order interval from 0 to a positive element in a partially ordered abelian group, the analogous interval in a partially ordered noncommutative group forms a pseudoeffect algebra (Example 2.1 below).

We review the definition and some of the notation for a pseudoeffect algebra $E$ in Section 2, and we study the center $\Gamma(E)$ of $E$ in Section 3. In Section 4, we focus attention on centrally orthocomplete pseudoeffect algebras and define the central cover of an element in a centrally orthocomplete pseudoeffect algebra. For the rest of the paper, we assume that $E$ is a centrally orthocomplete pseudoeffect algebra. The notion of a type-determining subset of $E$ is introduced in Section 5, where it is shown that type-determining subsets induce decompositions of $E$ into direct summands of various types. The paper ends with Section 6 where the important idea of a type-class of 
pseudoeffect algebras is introduced and a number of pertinent examples of type-classes and corresponding type-determining subsets of $E$ are presented.

\section{Pseudoeffect algebras}

To model algebraically the system of Hilbert-space effect operators, the notion of an effect algebra was introduced in [10]. By dropping the requirement of commutativity of the partially defined addition on an effect algebra, one arrives at the following definition [7, Definition 1.1].

A partial algebra $(E ;+, 0,1)$, where + is a partial binary operation and 0 and 1 are constants, is called a pseudoeffect algebra if and only if the following conditions hold for all $a, b, c \in E$.

(i) $a+b$ and $(a+b)+c$ exist if and only if $b+c$ and $a+(b+c)$ exist, and in this case $(a+b)+c=a+(b+c)$.

(ii) There is exactly one $d \in E$ and exactly one $e \in E$ such that $a+d=e+a=1$.

(iii) If $a+b$ exists, there are elements $d, e \in E$ such that $a+b=d+a=b+e$.

(iv) If $1+a$ or $a+1$ exists, then $a=0$.

Suppose that $E$ is a pseudoeffect algebra. If $a, b \in E$, define $a \leq b$ if and only if there exists an element $c \in E$ such that $a+c=b$; then $\leq$ is a partial ordering on $E$ such that $0 \leq a \leq 1$ for all $a \in E$. It is possible to show that $a \leq b$ if and only if $b=a+c=d+a$ for uniquely determined elements $c, d \in E$, and we write $c=: a / b$ and $d=: b \backslash a$. Then $(b \backslash a)+a=a+(a / b)=b$, and $a=(b \backslash a) / b=b \backslash(a / b)$. If $a \leq b \leq c$, then

$$
\begin{aligned}
(c \backslash a) \backslash(b \backslash a) & =c \backslash b ; & & (a / b) /(a / c)=b / c ; \\
(c \backslash b) /(c \backslash a) & =b \backslash a ; & & (a / c) \backslash(b / c)=a / b .
\end{aligned}
$$

We define $x^{-}:=1 \backslash x$ and $x^{\sim}:=x / 1$ for all $x \in E$. For a given element $e \in E$, we denote the order interval from 0 to $e$ by $E[0, e]:=\{x \in E: 0 \leq x \leq e\}$ and we define the partial binary operation $+_{e}$ on $E[0, e]$ as follows: for $f, g \in E[0, e], f+_{e} g$ exists if and only if $f+g$ exists in $E$ and $f+g \in E[0, e]$, in which case $f+e g=f+g$. Then $\left(E[0, e] ;+_{e}, 0, e\right)$ is a pseudoeffect algebra. Moreover, for all $x \in E[0, e]$, we have $x^{-e}:=e \backslash x, x^{\sim_{e}}:=x / e$, and $e=x^{-e}+x=x+x^{\sim_{e}}$.

For all $a, b \in E$, we write an existing least upper bound or greatest lower bound of $a$ and $b$ in the partially ordered set $E$ as $a \vee b$ or $a \wedge b$, respectively. Similarly, $\bigvee_{i \in I} e_{i}$ and $\bigwedge_{i \in I} e_{i}$ denote the least upper bound in $E$ and the greatest lower bound in $E$ of a family $\left(e_{i}\right)_{i \in I} \subseteq E$ if they exist. Elements $a, b \in E$ are disjoint if and only if $a \wedge b=0$. We say that $E$ is lattice-ordered if and only if $a \vee b$ and $a \wedge b$ exist in $E$ for all $a, b \in E$.

The following prototypical example will help to fix ideas.

EXAMPLE 2.1. Let $G$ be a partially ordered (not necessarily abelian) group, written additively, choose any element $0 \leq u \in G$, and let $G[0, u]=\{g \in G: 0 \leq g \leq u\}$. 
Then $(G[0, u] ;+, 0, u)$ is a pseudoeffect algebra under the restriction of the group operation + to $G[0, u]$. Clearly, the pseudoeffect algebra partial order on $G[0, u]$ is the restriction to $G[0, u]$ of the partial order on $G$. Moreover, for all $a, b \in G[0, u]$ such that $a \leq b$, we have $a / b=-a+b$ and $b \backslash a=b-a$.

Given elements $x_{1}, x_{2}, \ldots, x_{n}$ of a pseudoeffect algebra $E$, we define their orthosum $x_{1}+x_{2}+\cdots+x_{n}$ by recurrence: $x_{1}+\cdots+x_{n}$ exists if and only if both $x_{1}+\cdots+x_{n-1}$ and $\left(x_{1}+\cdots+x_{n-1}\right)+x_{n}$ exist, in which case we put

$$
x_{1}+\cdots+x_{n}:=\left(x_{1}+\cdots+x_{n-1}\right)+x_{n} .
$$

By associativity, we may omit parentheses, but the order of elements is important.

Let $E$ and $F$ be pseudoeffect algebras. A mapping $\phi: E \rightarrow F$ is a morphism of pseudoeffect algebras (pseudoeffect algebra morphism) if and only if $\phi\left(1_{E}\right)=1_{F}$, where $1_{E}$ and $1_{F}$ are the unit elements in $E$ and $F$, and $\phi(a)+\phi(b)$ exists whenever $a+b$ exists, with $\phi(a+b)=\phi(a)+\phi(b)$. A morphism is an isomorphism of pseudoeffect algebras (pseudoeffect algebra isomorphism) if and only if it is a bijection and $\phi^{-1}$ is also a morphism.

For more about the basic properties of pseudoeffect algebras see [7, 8].

\section{Central elements of pseudoeffect algebras}

For the rest of this paper, $(E ; 0,1,+)$ is a pseudoeffect algebra. Direct decompositions of $E$ are induced by the central elements of $E$, which are defined as follows.

Definition 3.1 [5, Definition 2.1]. An element $c$ of $E$ is said to be central if there exists an isomorphism ${ }^{1}$

$$
f_{c}: E \rightarrow E[0, c] \times E\left[0, c^{\sim}\right]
$$

such that $f_{c}(c)=(c, 0)$ and, for all $x \in E$, if $f_{c}(x)=\left(x_{1}, x_{2}\right)$, then $x=x_{1}+x_{2}$.

We denote by $\Gamma(E)$ the set of all central elements of $E$, and we refer to $\Gamma(E)$ as the center of $E$. Clearly, $0,1 \in \Gamma(E)$. In the next proposition, we gather together some properties of central elements (see [5, Propositions 2.2, 2.4, and 2.5] and [16]).

PROPOSITION 3.2. Let $c$ be a central element of $E$, and let $f_{c}$ be the corresponding mapping from Definition 3.1. Then the following properties hold for all $x, y, x_{1}$, $x_{2} \in E$.

(i) $f_{c}\left(c^{\sim}\right)=\left(0, c^{\sim}\right)$.

(ii) If $x \leq c$, then $f_{c}(x)=(x, 0)$.

(iii) $c \wedge c^{\sim}=0$.

(iv) If $y \leq c^{\sim}$ then $f_{c}(y)=(0, y)$.

\footnotetext{
${ }^{1}$ With coordinatewise operations, the cartesian product of pseudoeffect algebras is again a pseudoeffect algebra.
} 
(v) $c^{\sim}=c^{-}$.

(vi) $x \wedge c \in E, x \wedge c^{\sim} \in E$, and

$$
f_{c}(x)=\left(x \wedge c, x \wedge c^{\sim}\right) .
$$

(vii) If $f_{c}(x)=\left(x_{1}, x_{2}\right)$, then $x=x_{1} \vee x_{2}, x_{1} \wedge x_{2}=0$, and $x_{1}+x_{2}=x$.

(viii) The following are equivalent: $x \wedge c=0, x \leq c^{-}, x \leq c^{\sim}, c \leq x^{-}$, and $c \leq x^{\sim}$.

(ix) $c+c \in E$ implies $c=0$.

(x) Let $c_{1}, c_{2}, \ldots, c_{n} \in \Gamma(E), c_{i} \wedge c_{j}=0$ for $i \neq j$, and $c_{1}+c_{2}+\cdots+c_{n}=1$. Then

$$
x=x \wedge c_{1}+x \wedge c_{2}+\cdots+x \wedge c_{n}
$$

In view of Proposition 3.2(v), if $c \in \Gamma(E)$, then we shall write $c^{\prime}:=c^{-}=c^{\sim}$. Also, we say that elements $c, d \in \Gamma(E)$ are orthogonal if and only if $c \wedge d=0$.

TheOREM 3.3 [5, Theorem 2.3]. If $c, d \in \Gamma(E)$, then $c \wedge d$ exists in $E$ and belongs to $\Gamma(E)$, and $\Gamma(E)=\left(\Gamma(E) ; \wedge, \vee,{ }^{\prime}, 0,1\right)$ is a Boolean algebra.

If $c \in \Gamma(E)$, then the mapping $p_{c}: E \rightarrow E[0, c]$ defined by

$$
p_{c}(x):=x \wedge c \quad \forall x \in E
$$

is a morphism from $E$ onto $E[0, c]$ whose kernel is $E\left[0, c^{\prime}\right]$.

Proposition 3.4 [5, Proposition 2.6]. Let $x \in E$ and $c, d \in \Gamma(E)$. Then the following properties hold.

(i) $p_{c \wedge d}=p_{c} p_{d}=p_{d} p_{c}$.

(ii) $\quad c+d=c \vee d=d+c \quad$ and $\quad p_{c \vee d}(x)=p_{c}(x)+p_{d}(x)=p_{d}(x)+p_{c}(x) \quad$ if $c \wedge d=0$.

(iii) $c \backslash d=c \wedge d^{\prime}=d / c$ and $p_{c \wedge d^{\prime}}(x)=p_{c}(x) \backslash p_{d}(x)=p_{d}(x) / p_{c}(x)$ if $d \leq c$.

THEOREM 3.5 [5, Proposition 2.7]. Let $c_{1}, c_{2}, \ldots, c_{n} \in \Gamma(E)$ with $c_{i} \wedge c_{j}=0$ for $i \neq j$. Then the following properties hold.

(i) $\quad c:=\bigvee_{i=1}^{n} c_{i}=c_{1}+c_{2}+\cdots+c_{n} \in \Gamma(E)$ and, for all $x \in E$,

$$
x \wedge c=\bigvee_{i=1}^{n}\left(x \wedge c_{i}\right)=x \wedge c_{1}+\cdots+x \wedge c_{n}
$$

(ii) If $x_{i} \leq c_{i}$ for $i=1,2, \ldots, n$, then

$$
x_{1}+x_{2}+\cdots+x_{n}=x_{1} \vee x_{2} \vee \cdots \vee x_{n}=x_{i_{1}}+x_{i_{2}}+\cdots+x_{i_{n}},
$$

where $\left(i_{1}, i_{2}, \ldots, i_{n}\right)$ is any permutation of $(1,2, \ldots, n)$.

(iii) If $a_{1}, a_{2}, \ldots, a_{n} \in \Gamma(E)$ then, for all $x \in E$,

$$
x \wedge\left(\bigvee_{i=1}^{n} a_{i}\right)=\bigvee_{i=1}^{n}\left(x \wedge a_{i}\right) .
$$


THEOREM 3.6. Let $c_{1}, c_{2}, \ldots, c_{n}$ be pairwise orthogonal elements of $\Gamma(E)$ such that $c_{1}+c_{2}+\cdots+c_{n}=1$, let $X:=E\left[0, c_{1}\right] \times E\left[0, c_{2}\right] \times \cdots \times E\left[0, c_{n}\right]$, and define $\Phi: X \rightarrow E$ by $\Phi\left(e_{1}, e_{2}, \ldots, e_{n}\right):=e_{1}+e_{2}+\cdots+e_{n}=e_{1} \vee e_{2} \vee \cdots \vee e_{n}$ for all $\left(e_{1}, e_{2}, \ldots, e_{n}\right) \in X$. Then:

(i) $\Phi: X \rightarrow E$ is a pseudoeffect algebra isomorphism;

(ii) if $e \in E$, then $\Phi^{-1}(e)=\left(e \wedge c_{1}, e \wedge c_{2}, \ldots, e \wedge c_{n}\right)$.

PRoOF. If $\left(e_{1}, e_{2}, \ldots, e_{n}\right) \in X$, then $e_{1}+e_{2}+\cdots+e_{n}=e_{1} \vee e_{2} \vee \cdots \vee e_{n}$ by Theorem 3.5(ii). Clearly,

$$
\Phi(1)=\Phi\left(c_{1}, c_{2}, \ldots, c_{n}\right)=c_{1}+c_{2}+\cdots+c_{n}=1 .
$$

Assume that $\left(e_{1}, e_{2}, \ldots, e_{n}\right),\left(f_{1}, f_{2}, \ldots, f_{n}\right) \in X$ are such that

$$
\left(e_{1}, e_{2}, \ldots, e_{n}\right)+\left(f_{1}, f_{2}, \ldots, f_{n}\right)=\left(e_{1}+f_{1}, e_{2}+f_{2}, \ldots, e_{n}+f_{n}\right)
$$

exists in $X$. Then

$$
\begin{gathered}
\Phi\left(e_{1}, e_{2}, \ldots, e_{n}\right)=e_{1}+e_{2}+\cdots+e_{n}=e_{1} \vee \cdots \vee e_{n}, \\
\Phi\left(f_{1}, f_{2}, \ldots, f_{n}\right)=f_{1}+f_{2}+\cdots+f_{n}=f_{1} \vee \cdots \vee f_{n} .
\end{gathered}
$$

Now $e_{i}+f_{i}$ exists when $i=1,2, \ldots, n$, so $e_{i} \leq f_{i}^{-}$for $i=1,2, \ldots, n$, and when $i \neq j$, we have $e_{i} \leq c_{i}, f_{j} \leq c_{j}$, whence $e_{i} \leq c_{i} \leq c_{j}^{-} \leq f_{j}^{-}$. Then

$$
\Phi\left(e_{1}, \ldots, e_{n}\right)=e_{1} \vee e_{2} \vee \cdots \vee e_{n} \leq f_{1}^{-} \wedge f_{2}^{-} \wedge \cdots \wedge f_{n}^{-}=\Phi(f)^{-},
$$

so that $\Phi\left(\left(e_{1}, \ldots, e_{n}\right)\right)+\Phi\left(\left(f_{1}, \ldots, f_{n}\right)\right)$ exists. Moreover, by associativity and Theorem 3.5(ii),

$$
\begin{aligned}
\Phi\left(\left(e_{1}, \ldots, e_{n}\right)\right)+\Phi\left(\left(f_{1}, \ldots, f_{n}\right)\right) & =\left(e_{1}+e_{2}+\cdots+e_{n}\right)+\left(f_{1}+f_{2}+\cdots+f_{n}\right) \\
& =\left(e_{1}+f_{1}\right)+\left(e_{2}+f_{2}\right)+\cdots+\left(e_{n}+f_{n}\right) \\
& =\Phi\left(\left(e_{1}, e_{2}, \ldots, e_{n}\right)+\left(f_{1}, f_{2}, \ldots, f_{n}\right)\right) .
\end{aligned}
$$

This shows that $\Phi$ is additive. For each $e \in E$, define $\Psi: E \rightarrow X$ by

$$
\Psi(e):=\left(e \wedge c_{1}, e \wedge c_{2}, \ldots, e \wedge c_{n}\right)=\left(p_{c_{1}}(e), \ldots, p_{c_{n}}(e)\right) .
$$

Clearly, $\Psi(1)=1$ in $X$, and if $e+f$ exists, then $\Psi(e+f)=\Psi(e)+\Psi(f)$, since $p_{c_{i}}$ are morphisms for all $i$. Then $\Phi \circ \Psi(e)=e \wedge c_{1}+e \wedge c_{2}+\cdots+e \wedge c_{n}=e$ by Proposition 3.2(x). If $\left(e_{i}\right)_{i=1}^{n} \subseteq X$, then

$$
\Psi \circ \Phi\left(\left(e_{i}\right)_{i=1}^{n}\right)=\Psi\left(e_{1}+e_{2}+\cdots+e_{n}\right)=\left(p_{c_{i}}\left(e_{1}+\cdots+e_{n}\right)\right)_{i=1}^{n}=\left(e_{i}\right)_{i=1}^{n},
$$

since $p_{c_{i}}, i=1,2, \ldots, n$ is a morphism, and $e_{i} \leq c_{j}$ for $i=j$, while $e_{i} \leq c_{j}^{\prime}$ if $i \neq j$. It follows that $\Phi^{-1}=\Psi$, and $\Psi$ is a morphism, hence $\Phi$ is an isomorphism.

Theorem 3.7 [5, Proposition 2.8]. For all $c \in \Gamma(E), \Gamma(E[0, c])=\Gamma(E)[0, c]$. 
LEMma 3.8. Suppose that $e \in E,\left(f_{i}\right)_{i \in I} \subseteq E, e+f_{i}$ or $f_{i}+e$ exists for all $i \in I$, and $\bigvee_{i \in I} f_{i}$ exists in $E$. Then $\bigvee_{i \in I}\left(e+f_{i}\right)$ or $\bigvee_{i \in I}\left(f_{i}+e\right)$ respectively exists in $E$ and $e+\bigvee_{i \in I} f_{i}=\bigvee_{i \in I}\left(e+f_{i}\right)$ or $\bigvee_{i \in I} f_{i}+e=\bigvee_{i \in I}\left(f_{i}+e\right)$.

Proof. Let $f:=\bigvee_{i \in I} f_{i}$. Assume that $e+f_{i}$ exists for all $i \in I$. Then $f_{i} \leq e^{\sim}$ for all $i \in I$, so that $f \leq e^{\sim}$. Also $e+f_{i} \leq e+f$ for all $i \in I$. Suppose that $r \in E$ and $e+f_{i} \leq r$ for all $i \in I$. It suffices to prove that $e+f \leq r$. We have $e \leq e+f_{i} \leq$ $r=e+(e / r)$, whence $f_{i} \leq e / r$ for all $i \in I$, and it follows that $f \leq e / r$, hence $e+f \leq r$.

Now assume that $f_{i}+e$ exists for all $i \in I$. Then $f_{i} \leq e^{-}$, whence $f \leq e^{-}$. Then $f_{i}+e \leq f+e$, and let $r \in E$ be such that $f_{i}+e \leq r$ for all $i \in I$. Then $f_{i} \leq r \backslash e$ for all $i \in I$, whence $f \leq r \backslash e$, and this implies $f+e \leq r$.

Lemma 3.9. Suppose that $\phi: E \rightarrow E$ satisfies the conditions that $\phi(e)+f$ exists only if $e+\phi(f)$ exists, and $f+\phi(e)$ exists only if $\phi(f)+e$ exists for all $e, f \in E$. Then:

(i) $\phi$ is order-preserving;

(ii) if $\left(e_{i}\right)_{i \in I} \subseteq E$ and $e=\bigvee e_{i}$ exists in $E$, then $\bigvee \phi\left(e_{i}\right)$ exists in $E$ and $\phi(e)=$ $\bigvee_{i \in I} \phi\left(e_{i}\right)$.

PROOF. (i) Suppose that $e \leq f$. Then $f^{\sim} \leq e^{\sim}$, and as $\phi(f)+\phi(f)^{\sim}$ exists, $f+\phi\left(\phi(f)^{\sim}\right)$ exists, whence $\phi\left(\phi(f)^{\sim}\right) \leq f^{\sim} \leq e^{\sim}$, so $e+\phi\left(\phi(f)^{\sim}\right)$ exists and thus $\phi(e)+\phi(f)^{\sim}$ exists and finally $\phi(e) \leq \phi(f)$.

(ii) Assume the hypothesis of (ii). As $e_{i} \leq e$, it follows from (i) that $\phi\left(e_{i}\right) \leq \phi(e)$ for all $i \in I$. Suppose that $f \in E$ and $\phi\left(e_{i}\right) \leq f$ for all $i \in I$. Then $\phi\left(e_{i}\right)+f^{\sim}$ exists, which implies that $e_{i}+\phi\left(f^{\sim}\right)$ exists and so $e_{i} \leq\left(\phi\left(f^{\sim}\right)\right)^{-}$. It follows that $e \leq\left(\phi\left(f^{\sim}\right)\right)^{-}$whence $e+\phi\left(f^{\sim}\right)$ exists, so $\phi(e)+f^{\sim}$ exists and finally $\phi(e) \leq f$, proving (ii).

THEOREM 3.10. Let $c \in \Gamma(E)$ and let $\left(e_{i}\right)_{i \in I}$ be a family of elements of $E$. Then the following properties hold.

(i) If $\bigvee_{i \in I} e_{i}$ exists in $E$, then $c \wedge \bigvee_{i \in I} e_{i}=\bigvee_{i \in I}\left(c \wedge e_{i}\right)$

(ii) For every $e \in E, c=c \wedge e+c \wedge e^{\sim}$.

Proof. (i) Define $\phi: E \rightarrow E$ by $\phi(e):=c \wedge e$ for all $e \in E$. Suppose $e, f \in E$ and $\phi(e)+f$ exists. Then $c \wedge e \leq f^{-} \leq(c \wedge f)^{-}$and $c^{-} \wedge e \leq c^{-} \vee f^{-}=(c \wedge f)^{-}$. By Proposition 3.2(vi) and (vii), $e=(c \wedge e) \vee\left(c^{\prime} \wedge e\right) \leq(c \wedge f)^{-}$, and consequently, $e+\phi(f)$ exists. Now assume that $f+\phi(e)$ exists, then $c \wedge e \leq f^{\sim} \leq(c \wedge f)^{\sim}$, and $c^{\sim} \wedge e \leq c^{\sim} \vee f^{\sim}=(c \wedge f)^{\sim}$, and consequently $e=(c \wedge e) \vee\left(c^{\prime} \wedge e\right) \leq(c \wedge f)^{\sim}$, and so $\phi(f)+e$ exists. Therefore (i) follows from Lemma 3.9.

(ii) Put $e_{1}=e, e_{2}=e^{\sim}$. Then $e_{1}+e_{2}=1$ and

$$
c=p_{c}\left(e_{1}+e_{2}\right)=p_{c}\left(e_{1}\right)+p_{c}\left(e_{2}\right)=c \wedge e+c \wedge e^{\sim},
$$

as required. 
In the next theorem, we give an intrinsic characterization of central elements. (For a similar result, see [28].)

THEOREM 3.11. An element $c$ in a pseudoeffect algebra $E$ is central if and only if the following properties are satisfied.

(i) For all $a \in E$, there are $a_{1}, a_{2} \in E, a_{1} \leq c, a_{2} \leq c^{\sim}$ and $a=a_{1}+a_{2}$.

(ii) If $a+b$ is defined and either $a, b \leq c$ or $a, b \leq c^{\sim}$, then $a+b \leq c$ or $a+b \leq$ $c^{\sim}$ respectively.

(iii) If $x, y \in E, x \leq c, y \leq c^{\sim}$, then $x+y=y+x$.

PROOF. Observe first that properties (i)-(iii) above imply that $c^{\sim}=c^{-}$and $c \wedge c^{\sim}=0$. Indeed, by (iii), $1=c+c^{\sim}=c^{\sim}+c$, whence $c^{\sim}=c^{-}$. If $x \leq c, x \leq c^{\sim}$, then by (ii), $c+x \leq c$, whence $x=0$.

If $c$ is central, then property (i) follows by the definition of central elements.

To prove (ii), suppose that $a, b, c, a+b$ exist and $a, b \leq c$. Then $f_{c}(a)=(a, 0)$, $f_{c}(b)=(b, 0)$ and $f_{c}(a+b)=(a, 0)+(b, 0)=(a+b, 0)$, hence $a+b \leq c$. Part (iii) follows by Theorem 3.5(ii).

To prove the converse, assume that (i), (ii) and (iii) hold. By (i), we may define $f_{c}: E \rightarrow E[0, c] \times E\left[0, c^{\sim}\right]$ by $f_{c}(a)=\left(a_{1}, a_{2}\right)$ when $a=a_{1}+a_{2}$ where $a_{1} \leq c$ and $a_{2} \leq c^{\sim}$. We shall prove that $f_{c}$ satisfies Definition 3.1 in the following steps.

Step 1. Assume that $a=a_{1}+a_{2}=b_{1}+b_{2}$ where $a_{1}, b_{1} \leq c$ and $a_{2}, b_{2} \leq c^{\sim}$ are two decompositions of $a \in E$ by (i), and let $a^{\sim}=d_{1}+d_{2}$, where $d_{1} \leq c$ and $d_{2} \leq c^{\sim}$, be any decomposition of $a^{\sim}$. Then

$$
1=a+a^{\sim}=\left(a_{1}+a_{2}\right)+\left(d_{1}+d_{2}\right)=a_{1}+\left(a_{2}+d_{1}\right)+d_{2}
$$

by associativity. Since $a_{2} \leq c^{\sim}$ and $d_{1} \leq c$, we obtain by (iii) that $a_{2}+d_{1}=d_{1}+a_{2}$. Again by associativity, $1=\left(a_{1}+d_{1}\right)+\left(a_{2}+d_{2}\right)=c+c^{\sim}$, where $a_{1}+d_{1} \leq c$, $a_{2}+d_{2} \leq c^{\sim}$ by (ii). It follows that $a_{1}+d_{1}=c, a_{2}+d_{2}=c^{\sim}$, so $a_{1}=c \backslash d_{1}$, $a_{2}=c^{\sim} \backslash d_{2}$. Repeating this reasoning with $a_{1}, a_{2}$ replaced by $b_{1}, b_{2}$, we deduce that $a_{1}=b_{1}$ and $a_{2}=b_{2}$. This proves that $f_{c}$ is well defined.

Clearly, $f_{c}(c)=(c, 0)$ and if $x \in E$ with $f_{c}(x)=\left(x_{1}, x_{2}\right)$, then $x=x_{1}+x_{2}$.

If $f_{c}(a)=f_{c}(b)$, then $\left(a_{1}, a_{2}\right)=\left(b_{1}, b_{2}\right)$, which implies that $a_{1}=b_{1}$ and $a_{2}=b_{2}$, whence $a=b$. This shows that $f_{c}$ is injective.

Step 2. Let $a, b \in E$ be such that $a+b$ exists. Let $f_{c}(a)=\left(a_{1}, a_{2}\right), f_{c}(b)=\left(b_{1}, b_{2}\right)$, and $f_{c}(a+b)=\left(d_{1}, d_{2}\right)$. Then $a=a_{1}+a_{2}, b=b_{1}+b_{2}, a+b=d_{1}+d_{2}$. It follows that $\left(a_{1}+a_{2}\right)+\left(b_{1}+b_{2}\right)=a_{1}+b_{1}+a_{2}+b_{2}=d_{1}+d_{2}$, from (iii). By Step 1, we see that $d_{1}=a_{1}+b_{1}, d_{2}=a_{2}+b_{2}$. Therefore

$$
f_{c}(a+b)=\left(d_{1}, d_{2}\right)=\left(a_{1}+b_{1}, a_{2}+b_{2}\right)=\left(a_{1}, a_{2}\right)+\left(b_{1}, b_{2}\right)=f_{c}(a)+f_{c}(b) .
$$

This proves that $f_{c}$ is additive.

Step 3. Assume that $f_{c}(a)+f_{c}(b)$ exists in $E[0, c] \times E\left[0, c^{\sim}\right]$. Then

$$
\left(a_{1}, a_{2}\right)+\left(b_{1}, b_{2}\right)=\left(a_{1}+b_{1}, a_{2}+b_{2}\right),
$$


whence $a_{1}+b_{1}, a_{2}+b_{2}$ exist in $E$, and hence

$$
\left(a_{1}+b_{1}\right)+\left(a_{2}+b_{2}\right)=a_{1}+a_{2}+b_{1}+b_{2}=a+b .
$$

It follows that $a+b$ exists if and only if $f_{c}(a)+f_{c}(b)$ exists.

To prove surjectivity, take $(x, y) \in E[0, c] \times E\left[0, c^{\sim}\right]$ and define $z=x+y$. Then $f_{c}(z)=(x, y)$.

Steps 1-3 imply that $f_{c}$ is a bijection such that $f_{c}(a)+f_{c}(b)$ exists if and only if $a+b$ exists, and $f_{c}(a+b)=f_{c}(a)+f_{c}(b)$, hence it is an isomorphism.

LEMMA 3.12. If $p \in E$, then the following properties hold.

(i) $c \in \Gamma(E)$ implies that $p \wedge c \in \Gamma(E[0, p])$.

(ii) The mapping $c \mapsto p \wedge c$ for $c \in \Gamma(E)$ is a boolean homomorphism of $\Gamma(E)$ into the center $\Gamma(E[0, p])$ of $E[0, p])$.

PROOF. To prove (i), let $e \in E[0, p]$. Then

$$
e=e \wedge c+e \wedge c^{\prime}=e \wedge p \wedge c+e \wedge p \wedge c^{\prime} .
$$

Now $p \wedge c \leq p$ and $p=p \wedge c+(p \wedge c) / p=p \backslash(p \wedge c)+p \wedge c$. Comparing these expressions with $p=p \wedge c+p \wedge c^{\prime}=p \wedge c^{\prime}+p \wedge c$, we find that

$$
p \wedge c^{\prime}=(p \wedge c) / p=p \backslash(p \wedge c) .
$$

This implies that $(p \wedge c)^{\sim p}=(p \wedge c)^{-p}=p \wedge c^{\prime}$. Moreover, every $e \in E[0, p]$ has a decomposition $e=e_{1}+e_{2}$, where $e_{1} \leq p \wedge c$ and $e_{2} \leq p \wedge c^{\prime}=:(p \wedge c)^{\prime} p$. Suppose that $e, f \leq p \wedge c$ and $e+f$ exists in $E[0, p]$. Then $e+f \leq p$, and $e, f \leq c$ implies that $e+f \leq c$, so $e+f \leq p \wedge c$. The same argument holds if $e, f \leq(p \wedge c)^{\prime} p:=$ $p \wedge c^{\prime}$. If $x \leq p \wedge c$ and $y \leq p \wedge c^{\prime}$, then $x \leq c$ and $y \leq c^{\prime}$ imply that $x+y=y+x$. This proves that $p \wedge c \in \Gamma(E[0, p])$.

Part (ii) follows from Proposition 3.4.

\section{Centrally orthocomplete pseudoeffect algebras}

The centrally orthocomplete, pseudoeffect algebras, introduced and studied in this section, admit a very tractable theory of direct decomposition that is amenable to our subsequent work with type decompositions.

Definition 4.1. Two elements $p, q \in E$ are said to be $\Gamma$-orthogonal if and only if there are orthogonal central elements $c, d \in \Gamma(E)$ such that $p \leq c$ and $q \leq d$. A family $\left(e_{i}\right)_{i \in I}$ is $\Gamma$-orthogonal if and only if there is a pairwise orthogonal family of elements $\left(c_{i}\right)_{i \in I} \subseteq \Gamma(E)$ of central elements in $E$ such that $e_{i} \leq c_{i}$ for all $i \in I$.

Observe that, owing to Theorem 3.5(ii), if $e_{1}, e_{2}, \ldots, e_{n}$ are pairwise $\Gamma$-orthogonal elements, then their orthosum exists and does not depend on the order of its summands; moreover,

$$
\sum_{i=1,2, \ldots, n} e_{i}=e_{1}+e_{2}+\cdots+e_{n}=e_{1} \vee e_{2} \vee \cdots \vee e_{n}
$$


Definition 4.2. Let $\left(e_{i}\right)_{i \in I}$ be a $\Gamma$-orthogonal family in $E$ and let $\mathcal{F}$ be the collection of all finite subsets of the indexing set $I$. Then $\left(e_{i}\right)_{i \in I}$ is orthosummable if and only if

$$
\sum_{i \in I} e_{i}:=\bigvee_{F \in \mathcal{F}} \sum_{i \in F} e_{i}
$$

exists in $E$, in which case we refer to $\sum_{i \in I} e_{i}$ as the orthosum of the family. By definition, $E$ is a centrally orthocomplete pseudoeffect algebra if and only if every $\Gamma$-orthogonal family in $E$ is orthosummable.

LEMMA 4.3. The following properties hold.

(i) If $e$ and $f$ are $\Gamma$-orthogonal elements of $E$, then $e \leq f$ implies that $e=0$.

(ii) A family of central elements is $\Gamma$-orthogonal if and only if it is pairwise orthogonal, and this occurs if and only if it is pairwise disjoint.

(iii) Every finite $\Gamma$-orthogonal family in $E$ is orthosummable and its orthosum is its supremum in $E$.

(iv) An arbitrary $\Gamma$-orthogonal family in $E$ is orthosummable if and only if it has an orthosum, which occurs if and only if it has a supremum in E, and if it is orthosummable, then its orthosum coincides with its supremum.

(v) $E$ is a centrally orthocomplete pseudoeffect algebra if and only if every $\Gamma$-orthogonal family in $E$ has a supremum in $E$.

Proof. We prove (i). If $e, f \in E$ and $c, d \in \Gamma(E)$ such that $e \leq c$ and $f \leq d \leq c^{\prime}$, then $e \leq f$ implies that $e \leq c \wedge c^{\prime}=0$ by Proposition 3.2(iii) and (v).

Part (ii) follows directly from the definitions of $\Gamma$-orthogonality and orthogonality of central elements.

Part (iii) follows from Theorem 3.5(ii).

Part (iv) follows from (iii) and the definition of the orthosum.

Part (v) follows from (iv).

In the rest of this section, we assume that $E$ is a centrally orthocomplete pseudoeffect algebra.

THEOREM 4.4. Let $\left(c_{i}\right)_{i \in I}$ be a pairwise orthogonal family of elements in $\Gamma(E)$, and let $\left(e_{i}\right)_{i \in I}$ and $\left(f_{i}\right)_{i \in I}$ be families in $E$ such that $e_{i}, f_{i} \leq c_{i}$ and $e_{i}+f_{i}$ exists for all $i \in I$. Then the following properties hold.

(i) $\quad c:=\sum_{i \in I} c_{i}=\bigvee_{i \in I} c_{i}, \quad e:=\sum_{i \in I} e_{i}=\bigvee_{i \in I} e_{i} \leq c \quad$ and $f:=\sum_{i \in I} f_{i}=$ $\bigvee_{i \in I} f_{i} \leq c$; furthermore, $e+f$ exists.

(ii) $e+f=\sum_{i \in I}\left(e_{i}+f_{i}\right)=\bigvee_{i \in I}\left(e_{i}+f_{i}\right) \leq c$.

PROOF. Part (i) follows from parts (ii) and (iv) of Lemma 4.3. For instance, the existence of $e+f$ is proved as follows. As $e_{i}+f_{i}$ exists for all $i \in I$, we have $e_{i} \leq$ $f_{i}^{-}$. If $i \neq j$, then $e_{i} \leq c_{i}, f_{j} \leq c_{j}$, and $c_{i} \wedge c_{j}=0$, so $e_{i}+f_{j}$ exists and $e_{i} \leq f_{j}^{-}$. Then $e=\bigvee_{i \in I} e_{i} \leq f_{j}^{-}$for all $j \in I$, whence $e \leq \bigwedge_{j \in I} f_{j}^{-}=\left(\bigvee_{j \in I} f_{j}\right)^{-}=f^{-}$, so $e+f$ exists. 
To prove (ii), observe that if $i \in I$, then $e_{i}, f_{i} \leq c_{i}$ implies that $e_{i}+f_{i} \leq c_{i}$ by Theorem 3.11(ii). Hence the family $\left(e_{i}+f_{i}\right)_{i \in I}$ is $\Gamma$-orthogonal in $E$, so by Lemma 4.3(iv) and (v),

$$
\sum_{i \in I}\left(e_{i}+f_{i}\right)=\bigvee_{i \in I}\left(e_{i}+f_{i}\right) \leq \bigvee_{i \in I} c_{i}=c .
$$

By Lemma 3.8,

$$
e+f=\left(\bigvee_{s \in I} e_{s}\right)+f=\bigvee_{s \in I}\left(e_{s}+f\right)
$$

and for each $s \in I$, we observe that $e_{s}+f=e_{s}+\bigvee_{t \in I} f_{t}=\bigvee_{t \in I}\left(e_{s}+f_{t}\right)$, and so

$$
\bigvee_{i \in I}\left(e_{i}+f_{i}\right) \leq \bigvee_{s, t \in I}\left(e_{s}+f_{t}\right)=e+f
$$

Suppose that $s, t \in I$. If $s=t$, then $e_{s}+f_{t}=e_{s}+f_{s} \leq \bigvee_{i \in I}\left(e_{i}+f_{i}\right)$. If $s \neq t$, then we see that

$$
e_{s}+f_{t} \leq\left(e_{s}+f_{s}\right)+\left(e_{t}+f_{t}\right)=\left(e_{s}+f_{s}\right) \vee\left(e_{t}+f_{t}\right) .
$$

Consequently,

$$
e+f=\bigvee_{s, t \in I}\left(e_{s}+f_{t}\right) \leq \bigvee_{i \in I}\left(e_{i}+f_{i}\right)
$$

Combining the results obtained above, we get (ii).

COROLlaRY 4.5. Let $\left(c_{i}\right)_{i \in I}$ be a pairwise orthogonal family of elements in $\Gamma(E)$ and let $d \in E$. Put $c:=\bigvee_{i \in I} c_{i}, e:=\bigvee_{i \in I}\left(d \wedge c_{i}\right)$, and $f:=\bigvee_{i \in I}\left(d^{\sim} \wedge c_{i}\right)$. Then the following properties hold.

(i) $e \leq d, f \leq d^{\sim}$, and $c=e+f$.

(ii) If $d \in E[0, c]$, then $d=\sum_{i \in I}\left(d \wedge c_{i}\right)=\bigvee_{i \in I}\left(d \wedge c_{i}\right)$.

PROOF. In Theorem 4.4, let $e_{i}:=d \wedge c_{i}$ and $f_{i}:=d^{\sim} \wedge c_{i}$.

(i) As $e_{i} \leq d$ and $f_{i} \leq d^{\sim}$ for all $i \in I$, we get $e=\bigvee_{i \in I} e_{i} \leq d$ and $f=\bigvee_{i \in I} f_{i} \leq d^{\sim}$. By Theorem 3.10(iii), $e_{i}+f_{i}=c_{i}$ for all $i \in I$, whence by Theorem 4.4(ii), $e+f=\bigvee_{i \in I}\left(e_{i}+f_{i}\right)=\bigvee_{i \in I} c_{i}=c$.

(ii) Assume that $d \in E[0, c]$. Then $e \leq d \leq c$ by (i). Thus $e \leq\left(d^{\sim}\right)^{-}$, hence $e+d^{\sim}$ exists, and $e+d^{\sim}=\bigvee_{i \in I}\left(e_{i}+d^{\sim}\right)$ by Lemma 3.8. As $c_{i} \in \Gamma(E)$,

$$
\begin{aligned}
e_{i}+d^{\sim} & =\left(d \wedge c_{i}\right)+d^{\sim}=\left(d \wedge c_{i}\right)+\left(d^{\sim} \wedge c_{i}\right)+\left(d^{\sim} \wedge c_{i}^{\sim}\right)=c_{i}+\left(d^{\sim} \wedge c_{i}^{\sim}\right) \\
& =c_{i} \vee\left(d^{\sim} \wedge c_{i}^{\sim}\right)=c_{i} \vee\left(d^{\sim} \wedge c_{i}\right) \vee\left(d^{\sim} \wedge c_{i}^{\sim}\right)=c_{i} \vee d^{\sim},
\end{aligned}
$$

so

$$
e+d^{\sim}=\bigvee_{i \in I}\left(d^{\sim} \vee c_{i}\right) \geq \bigvee_{i \in I}\left(c^{\sim} \vee c_{i}\right)=c^{\sim} \vee c=1=e+e^{\sim}
$$

By cancellation, $d^{\sim} \geq e^{\sim}$, whence $d \leq e$, and we have $e=d$. 
THEOREM 4.6. Let $\left(c_{i}\right)_{i \in I}$ be a pairwise orthogonal family of central elements, and let $c:=\bigvee_{i \in I} c_{i}$. Then $c \in \Gamma(E)$, and $\Gamma(E)$ is a complete boolean algebra. Furthermore, for each $e \in E$ there is a smallest element $d \in \Gamma(E)$ such that $e \leq d$.

PROOF. First, we have to prove properties (i)-(iii) of Theorem 3.11 for $c$.

To prove (i), let $d \in E$. By Corollary 4.5, $c=e+f, e \leq d, f \leq d^{\sim}$. Consequently, $d=e+e / d$, and $e / d=\bigvee_{i \in I}\left(d \wedge c_{i}\right) / d \leq d \wedge c_{i} / d$ for all $i \in I$. Let $x \in E$ be such that $x \leq d \wedge c_{i} / d$ for all $i \in I$. Then $d \wedge c_{i}+x \leq d$, hence $d \wedge c_{i} \leq d \backslash x$. It follows that $\bigvee_{i \in I}\left(d \wedge c_{i}\right) \leq d \backslash x$, and therefore $x \leq \bigvee_{i \in I}\left(d \wedge c_{i}\right) / d$. This proves that

$$
\bigvee_{i \in I}\left(d \wedge c_{i}\right) / d=\bigwedge_{i \in I}\left(d \wedge c_{i} / d\right)=\bigwedge_{i \in I} d \wedge c_{i}^{\sim} \leq \bigwedge_{i \in I} c_{i}^{\sim}=\left(\bigvee_{i \in I} c_{i}\right)^{\sim}=c^{\sim} .
$$

Finally, $d=e+e / d, e \leq c, e / d \leq c^{\sim}$.

Next, let $e, f \leq c$ and suppose that $e+f$ exists. Then $e_{i}:=e \wedge c_{i} \leq c_{i}, f_{i}:=$ $f \wedge c_{i} \leq c_{i},\left(e_{i}\right)_{i \in I}$ and $\left(f_{i}\right)_{i \in I}$ are $\Gamma$-orthogonal, and $e_{i}+f_{i}$ exists for all $i \in I$. By Theorem 4.4,e $=\bigvee_{i \in I} e_{i}, f=\bigvee_{i \in I} f_{i}$, and $e+f=\bigvee_{i \in I}\left(e_{i}+f_{i}\right) \leq c$. Let $e, f \leq c^{\sim}$ and suppose that $e+f$ exists. From $c^{\sim}=\left(\bigvee_{i \in I} c_{i}\right)^{\sim}=\bigwedge_{i \in I} c_{i}^{\sim}$ we obtain that $e, f \leq c_{i}^{\sim}$ for all $i \in I$, and since $c_{i}$ is central, $e+f \leq c_{i}^{\sim}$ for all $i \in I$. It follows that $e+f \leq \bigwedge_{i \in I} c_{i}^{\sim}=c^{\sim}$, and (ii) holds.

Third, let $x, y \in E, x \leq c, y \leq c^{\sim}$. Then $x \wedge c_{i} \leq c_{i}, y \leq c^{\sim} \leq c_{i}^{\sim}$ for all $i \in I$, and $x=\bigvee_{i \in I} x \wedge c_{i}$ by Theorem 3.10. Since $c_{i}$ is central, $x \wedge c_{i}+y=y+x \wedge c_{i}$, and by Lemma 3.8,

$$
x+y=\bigvee_{i \in I}\left(x \wedge c_{i}+y\right)=\bigvee_{i \in I}\left(y+x \wedge c_{i}\right)=y+x .
$$

This proves (iii).

Therefore $c \in \Gamma(E)$, and by [26, Section 20.1], $\Gamma(E)$ is a complete boolean algebra.

To prove the second part of the theorem, we put $f=e^{\sim}$. Using Zorn's lemma we choose a maximal pairwise orthogonal family $\left(c_{i}\right)_{i \in I}$ in $\Gamma(E) \cap E[0, f]$. As $c_{i} \leq f$ for all $i \in I$, we have $c:=\bigvee_{i \in I} c_{i} \leq f$, and $c \in \Gamma(E)$ by part (i) of this proof. Then $d:=c^{-}=\bigwedge_{i \in I} c_{i}^{-}$and $e=f^{-} \leq c^{-}=d \in \Gamma(E)$. To show that $d$ is the smallest element in $\Gamma(E)$ such that $e \leq d$, let $e \leq k \in \Gamma(E)$. Then $k^{\sim} \leq e^{\sim}=f$, so $k^{\sim} \wedge d \in \Gamma(E) \cap E[0, f]$. Then $k^{\sim} \wedge d \leq d=c^{-} \leq c_{i}^{-}=c_{i}^{\prime}$ for all $i \in I$, hence $k^{\sim} \wedge d$ is orthogonal to all $c_{i}$, and so $k^{\sim} \wedge d=k^{\prime} \wedge d=0$ by maximality of $\left(c_{i}\right)_{i \in I}$. Since $k, d \in \Gamma(E), d \leq k$, as required.

Definition 4.7. If $e \in E$, then the smallest element $d \in \Gamma(E)$ such that $e \leq d$ (as in Theorem 4.6) is called the central cover of $e$, and we shall denote it by $\gamma e:=d$. The mapping $e \mapsto \gamma e$ is said to be the central cover mapping.

The hull mappings featured in the original work of Loomis on dimension lattices [21] were generalized to effect algebras in [11, 12]. In the following definition, we further extend the notion of a hull mapping to pseudoeffect algebras. 
DeFinition 4.8. A mapping $\eta: E \rightarrow \Gamma(E)$ such that $\eta 0=0, \quad e \leq \eta e$ and $\eta(e \wedge \eta f)=\eta e \wedge \eta f$ for all $e, f \in E$ is called a hull mapping on $E$.

THEOREM 4.9. The central cover mapping $\gamma: E \rightarrow \Gamma(E)$ is a surjective hull mapping $^{1}$ on $E$.

PROOF. Obviously, $\gamma 0=0$ and $e \leq \gamma e$ for all $e \in E$. Let $e, f \in E$ and put $c:=\gamma f$. We have to prove that $\gamma(e \wedge c)=\gamma e \wedge c$. Since $e \leq \gamma e$, we have $e \wedge c \leq \gamma e \wedge c$, and so $\gamma(e \wedge c) \leq \gamma e \wedge c$. Since $c \in \Gamma(E)$, we have $e=(e \wedge c) \vee\left(e \wedge c^{\prime}\right) \leq \gamma(e \wedge c)$ $\vee c^{\prime} \in \Gamma(E)$, whence $\gamma e \leq \gamma(e \wedge c) \vee c^{\prime}$. It follows that $\gamma e \wedge c \leq \gamma(e \wedge c) \wedge c \leq$ $\gamma(e \wedge c)$, as desired. Since $\gamma(\gamma e)=\gamma(1 \wedge \gamma e)=\gamma 1 \wedge \gamma e=\gamma e$, we obtain that $\gamma E:=\{\gamma e: e \in E\}=\Gamma(E)$.

LEMmA 4.10. Suppose that $\left(p_{i}\right)_{i \in I} \subseteq E$ is a $\Gamma$-orthogonal family in $E$. Let $p:=$ $\bigvee_{i \in I} p_{i}$, and let $c_{i}:=\gamma p_{i}$ for all $i \in I$ with $c=\bigvee_{i \in I} c_{i}$. Then the following properties hold.

(i) $p \leq \gamma p=c \in \Gamma(E)$.

(ii) $p \wedge c_{i}=p_{i}$ for all $i \in I$.

(iii) If $e \in E[0, p]$, then $e \wedge c_{i}=e \wedge p_{i}$ for all $i \in I$ and $e=\bigvee_{i \in I}\left(e \wedge p_{i}\right)$.

PROOF. Since $\left(p_{i}\right)_{i \in I}$ is a $\Gamma$-orthogonal family, $\left(c_{i}\right)_{i \in I}$ is an orthogonal family in $\Gamma(E)$, so $p$ and $c$ are well defined. Since $p_{i} \leq p$ for all $i \in I$, we have $\bigvee_{i \in I} \gamma p_{i}=$ $c \leq \gamma p$. On the other hand, $p_{i} \leq \gamma p_{i} \leq c$ implies $\gamma p \leq c$. This proves (i).

Suppose that $i, j \in I$. If $i=j$, then $p_{i} \wedge c_{i}=p_{i} \wedge \gamma p_{i}=p_{i}$; and if $i \neq j$, then $c_{i} \wedge c_{j}=0$, so $c_{i} \wedge p_{j}=0$. Therefore

$$
p \wedge c_{i}=\left(\bigvee_{j \in I} p_{j}\right) \wedge c_{i}=\bigvee_{j \in I}\left(p_{j} \wedge c_{i}\right)=p_{i}
$$

by Theorem 3.10(i), which proves (ii).

To prove (iii), suppose $e \in E[0, p]$. Then for each $i \in I, e \wedge c_{i}=e \wedge p \wedge c_{i}=$ $e \wedge p_{i}$ by (ii). Thus by Corollary 4.5(ii), $e=e \wedge c=\bigvee_{i \in I}\left(e \wedge c_{i}\right)=\bigvee_{i \in I}\left(e \wedge p_{i}\right)$.

The following theorem extends Theorem 3.6 in the setting of centrally orthocomplete pseudoeffect algebras. Since the proof is analogous to [11, Theorem 6.14], we omit it.

THEOREM 4.11. Let $\left(p_{i}\right)_{i \in I} \subseteq E$ be a $\Gamma$-orthogonal family in $E$, let $p:=\sum_{i \in I} p_{i}=$ $\bigvee_{i \in I} p_{i}$, and let $X:=\prod_{i \in} E\left[0, p_{i}\right]$. Define the mapping $\Phi: X \rightarrow E[0, p]$ by

$$
\Phi\left(\left(e_{i}\right)_{i \in I}\right):=\sum_{i \in I} e_{i}=\bigvee_{i \in I} e_{i} \text { for every }\left(e_{i}\right)_{i \in I} \in X .
$$

Then $\Phi$ is a pseudoeffect algebra isomorphism of $X$ onto $E[0, p]$ and

$$
\Phi^{-1}(e):=\left(e \wedge \gamma p_{i}\right)_{i \in I} \text { for all } e \in E[0, p] .
$$

\footnotetext{
${ }^{1}$ In [11], a surjective hull mapping from an effect algebra $E$ onto $\Gamma(E)$ (which is unique if it exists) is called a discrete hull mapping.
} 


\section{Type-determining sets}

The assumption that $E$ is a centrally orthocomplete pseudoeffect algebra remains in force.

Our definition of a type-determining subset of $E$ will depend on certain closure operators on subsets of $E$. As usual, a closure operator on the set of all subsets $Q$ of $E$ is a mapping $Q \mapsto Q^{c}$ such that $Q \subseteq Q^{c},\left(Q^{c}\right)^{c}=Q^{c}$ and $Q^{c} \subseteq R^{c}$ if $Q \subseteq R$ for all $Q, R \subseteq E$. A subset $Q$ is said to be closed (with respect to ${ }^{c}$ ) if and only if $Q^{c}=Q$. The intersection of closed subsets is necessarily closed. Generalizing the analogous notions for effect algebras in [13], we introduce the four closure operators $Q \mapsto[Q], Q \mapsto Q^{\gamma}, Q \mapsto Q^{\downarrow}$, and $Q \mapsto Q^{\prime \prime}$, where:

(i) $[Q]$ is the set of all suprema of $\Gamma$-orthogonal families of elements of $Q$, and we define $[\emptyset]=\{0\}$;

(ii) $Q^{\gamma}:=\{q \wedge c: q \in Q, c \in \Gamma(E)\}$;

(iii) $Q^{\downarrow}:=\bigcup_{q \in Q} E[0, q]$;

(iv) $Q^{\prime}:=\{e \in E: q \wedge e=0 \forall q \in Q\}$;

(v) $Q^{\prime \prime}:=\left(Q^{\prime}\right)^{\prime}$.

Definition 5.1. We say that a subset $K \subseteq E$ is type-determining if and only if $K=[K]=K^{\gamma}$, and that $K$ is strongly type-determining if and only if $K=[K]=K^{\downarrow}$.

Clearly, the intersection of type-determining or strongly type-determining subsets of $E$ is again type-determining or strongly type-determining.

THEOREM 5.2. Let $Q \subseteq E$. Then the following properties hold.

(i) $\left[Q^{\gamma}\right]$ is the smallest type-determining subset of $E$ containing $Q$.

(ii) $\left[Q^{\downarrow}\right]$ is the smallest strongly type-determining subset of $E$ containing $Q$.

(iii) $Q^{\prime}$ and $Q^{\prime \prime}$ are strongly type-determining subsets of $E$.

(iv) $Q^{\prime}=\left[Q^{\gamma}\right]^{\prime}=\left[Q^{\downarrow}\right]^{\prime}$.

Proof. Obviously, $Q \subseteq\left[Q^{\gamma}\right]$ and if $K$ is type-determining and $Q \subseteq K$, then $\left[Q^{\gamma}\right] \subseteq K$. Also, [[ $\left.\left.Q^{\gamma}\right]\right] \subseteq\left[Q^{\gamma}\right]$, so to prove (i) it suffices to show that $\left[Q^{\gamma}\right]^{\gamma} \subseteq\left[Q^{\gamma}\right]$. Let $e \in\left[Q^{\gamma}\right]^{\gamma}$, then there exist $d \in \Gamma(E)$ and $p \in\left[Q^{\gamma}\right]$ such that $e=p \wedge d$. As $p \in\left[Q^{\gamma}\right]$, there is a $\Gamma$-orthogonal family $\left(p_{i}\right)_{i \in I} \subseteq Q^{\gamma}$ with $p=\bigvee_{i \in I} p_{i}$, and for each $i \in I$, we can write $p_{i}=q_{i} \wedge d_{i}$ with $q_{i} \in Q$ and $d_{i} \in \Gamma(E)$. Since $e \leq p$, by Lemma 4.10(iii), $e \wedge p_{i}$ exists for all $i \in I$; moreover, $e \wedge p_{i}=p \wedge d \wedge p_{i}=$ $p_{i} \wedge d=q_{i} \wedge d_{i} \wedge d$. As $d_{i} \wedge d \in \Gamma(E)$, it follows that $e \wedge p_{i} \in Q^{\gamma}$ for all $i \in I$, and the family $\left(e \wedge p_{i}\right)_{i \in I}$ is $\gamma$-orthogonal. Consequently, by Lemma 4.10(iii), $e=\bigvee_{i \in I}\left(e \wedge p_{i}\right) \in\left[Q^{\gamma}\right]$. This proves (i).

The proof of (ii) is quite similar to the proof of (i), and we omit it.

To prove (iii), let $e \in Q^{\prime}$ and $f \leq e$. Then $e \wedge q=0$ for all $q \in Q$, whence $f \wedge q=0$ for all $q \in Q$, hence $f \in Q^{\prime}$, so that $Q=Q^{\downarrow}$. Let $\left(p_{i}\right)_{i \in I} \subseteq Q^{\prime}$ be a $\Gamma$ orthogonal family, and $p=\bigvee_{i \in p_{i}}$. Then $q \wedge p_{i}=0$ for all $q \in Q$ and all $i \in I$, and since $q \wedge p \leq p$, by Lemma 4.10(iii), $p \wedge q=\bigvee_{i \in I} p \wedge q \wedge p_{i}=0$, hence $p \in Q^{\prime}$. It 
follows that $Q^{\prime}=\left[Q^{\prime}\right]$, and $Q^{\prime}$ is strongly type-determining. As $Q^{\prime \prime}=\left(Q^{\prime}\right)^{\prime}$, it follows that $Q^{\prime \prime}$ is strongly type-determining.

To prove (iv), observe that $Q \subseteq\left[Q^{\gamma}\right] \subseteq\left[Q^{\downarrow}\right]$ implies $\left[Q^{\downarrow}\right]^{\prime} \subseteq\left[Q^{\gamma}\right]^{\prime} \subseteq Q^{\prime}$. Let $e \in Q^{\prime}$, and let $\left(p_{i}\right)_{i \in I}$ be a $\Gamma$-orthogonal family of elements in $Q^{\downarrow}$ with $p=$ $\bigvee_{i \in I} p_{i}$. Then each $p_{i} \leq q_{i}$ for some $q_{i} \in Q$, and $e \wedge p_{i} \leq e \wedge q_{i}=0$ for all $i \in I$. By Lemma 4.10(iii), $e \wedge p=\bigvee_{i \in I} e \wedge p \wedge p_{i}=0$, which shows that $e \in\left[Q^{\downarrow}\right]^{\prime}$, proving (iv).

THEOREM 5.3. Let $K \subseteq E$ be a type-determining set. Then the following properties hold.

(i) $\quad K \cap \gamma K=K \cap \Gamma(E) \subseteq \gamma K \subseteq \Gamma(E)$.

(ii) There exists $c \in \Gamma(E)$ such that $\gamma K \subseteq \Gamma(E)[0, c]$.

(iii) There exists $d \in \Gamma(E)$ such that $K \cap \gamma K=\Gamma(E)[0, d]$.

PROOF. We omit the proof since it is analogous to that of [13, Theorem 4.5].

Obviously, for every $c \in \Gamma(E)$, the central interval $\Gamma(E)[0, c]=\Gamma(E) \cap E[0, c]$ is a type-determining subset of $E$.

COROllary 5.4. If $K$ is a type-determining subset of $E$, then so are $\gamma K$ and $K \cap \gamma K$.

Definition 5.5. Let $K$ be a type-determining subset of $E$. The (unique) element $c \in \gamma K$ such that $\gamma K=\Gamma(E)[0, c]$ (Theorem 5.3(ii)) is denoted by $c_{K}$ and is called the type-cover of $K$. The type-cover $c_{K \cap \gamma K}$ of the type-determining set $K \cap \gamma K$ is called the restricted type-cover of $K$.

The following definition is analogous to [13, Definition 5.1]. The terminology is borrowed from [27, pp. 28-29].

DEFINITION 5.6. Let $K$ be a type-determining subset of the centrally orthocomplete pseudoeffect algebra $E$ and let $c \in \Gamma(E)$.

(i) $c$ is type- $K$ if and only if $c \in K$.

(ii) $c$ is locally type- $K$ if and only if $c \in \gamma K$.

(iii) $c$ is purely non- $K$ if and only if no nonzero subelement of $c$ belongs to $K$.

(iv) $c$ is properly non- $K$ if and only if no nonzero central subelement of $c$ belongs to $K$.

If $c \in \Gamma(E)$ and $c$ is type- $K$ or locally type- $K$, and so on, we shall also say that the direct summand $E[0, c]$ of $E$ is type- $K$ or locally type- $K$, and so on.

The proof of the next theorem is omitted since it is the same as the proof of [13, Theorem 5.2].

THEOREM 5.7. Let $K$ be a type-determining subset of $E$ and let $c \in \Gamma(E)$. Then the following properties hold.

(i) $\quad c$ is type- $K$ if and only if $\Gamma(E)[0, c] \subseteq K \cap \gamma K$, or equivalently, $c \leq c_{K \cap \gamma K}$.

(ii) If $K$ is strongly type-determining, then $c$ is type- $K$ if and only if $E[0, c] \subseteq K$. 
(iii) $c$ is locally type- $K$ if and only if $\Gamma(E)[0, c] \subseteq \gamma K$, or equivalently, $c \leq c_{K}$.

(iv) $c$ is purely non- $K$ if and only if $K \cap E[0, c]=\{0\}$, or equivalently, $c \leq\left(c_{K}\right)^{\prime}$.

(v) $c$ is properly non- $K$ if and only if $K \cap \Gamma(E)[0, c]=\{0\}$, or equivalently, $c \leq$ $\left(c_{K \cap \gamma K}\right)^{\prime}$.

(vi) $c$ is both locally type- $K$ and properly non- $K$ if and only if $c \leq c_{K} \wedge\left(c_{K \cap \gamma K}\right)^{\prime}$.

LEMMA 5.8. If $K$ is a type-determining subset of $E$, then $c_{K^{\prime} \cap \gamma\left(K^{\prime}\right)}=\left(c_{K}\right)^{\prime}$.

Proof. We must prove that $K^{\prime} \cap \gamma\left(K^{\prime}\right)=\Gamma(E)\left[0,\left(c_{K}\right)^{\prime}\right] . \quad$ As $K^{\prime} \cap \gamma\left(K^{\prime}\right)=$ $K^{\prime} \cap \Gamma(E)$, it suffices to prove that, for any $c \in \Gamma(E)$, we have $c \in K^{\prime}$ if and only if $c \leq\left(c_{K}\right)^{\prime}$, the latter inequality being equivalent to $c \wedge c_{K}=0$.

Let $c \in \Gamma(E)$. Suppose that $c \in K^{\prime}$ and let $k^{*} \in K$ be such that $c_{K}=\gamma k^{*}$. Then $c \wedge k^{*}=0$, whence $c \wedge c_{K}=\gamma\left(c \wedge k^{*}\right)=0$. Conversely, suppose that $c \wedge c_{K}=0$ and let $k \in K$. Then, as $\gamma k \leq c_{K}$, it follows that $\gamma(c \wedge k)=c \wedge \gamma k=0$, whence $c \wedge k=0$, so $c \in K^{\prime}$.

THEOREM 5.9. Let $K$ be a type-determining subset of $E$. Then there exist unique pairwise orthogonal $c_{1}, c_{2}, c_{3} \in \Gamma(E)$ such that $c_{1}+c_{2}+c_{3}=1$;

$$
E=E\left[0, c_{1}\right] \oplus E\left[0, c_{2}\right] \oplus E\left[0, c_{3}\right] ;
$$

$c_{1}$ is type- $K ; c_{2}$ is locally type- $K$, but properly non- $K$; and $c_{3}$ is purely non- $K$. Moreover, $c_{1}=c_{K \cap \gamma K}, c_{2}=c_{K} \wedge\left(c_{K \cap \gamma k}\right)^{\prime}, c_{3}=\left(c_{K}\right)^{\prime}$,

$$
K \cap \gamma K=\Gamma(E)\left[0, c_{1}\right], \quad K \subseteq E\left[0, c_{1}+c_{2}\right], \quad \Gamma(E)\left[0, c_{2}+c_{3}\right] \cap K=\{0\} .
$$

ProOF. Put $c_{1}:=c_{K \cap \gamma K}, c_{2}:=c_{K} \wedge\left(c_{K \cap \gamma K}\right)^{\prime}$, and $c_{3}:=\left(c_{K}\right)^{\prime}$. As $c_{K \cap \gamma K} \leq c_{K}$, we have $c_{1}+c_{2}+c_{3}=1, c_{1}+c_{2}=c_{K}$, and $c_{2}+c_{3}=\left(c_{K \cap \gamma K}\right)^{\prime}$. Thus, by part (i) of Theorem 5.7(i), $c_{1}$ is of type- $K$; by part (v) of Theorem 5.7, $c_{2}$ is locally type- $K$ and properly non- $K$; and by part (iv) of Theorem $5.7, c_{3}$ is purely non- $K$.

To prove uniqueness, suppose that $c_{1}, c_{2}$ and $c_{3}$ satisfy the conditions in the first part of the theorem. Then $c_{1}+c_{2}$ is locally type- $K$, hence $c_{1}+c_{2} \leq c_{K}$, and $c_{3}$ is purely non- $K$, hence $c_{3} \leq\left(c_{K}\right)^{\prime}$ by Theorem 5.7(iii) and (iv). Now $c_{1}+c_{2}=c_{K}$ and $c_{3}=\left(c_{K}\right)^{\prime}$, since $c_{1}+c_{2}+c_{3}=1=c_{K}+\left(c_{K}\right)^{\prime}$. Moreover, $c_{1}$ is type- $K$, hence $c_{1} \leq c_{K \cap \gamma K}$, and $c_{2}$ is locally type- $K$ but properly non- $K$, hence $c_{2} \leq c_{K} \wedge\left(c_{K \cap \gamma K}\right)^{\prime}$. Since $c_{1}+c_{2}=c_{K}=c_{K \cap \gamma K}+c_{K} \wedge\left(c_{K \cap \gamma K}\right)^{\prime}$, we obtain $c_{1}=c_{K \cap \gamma K}, \quad c_{2}=$ $c_{K} \wedge\left(c_{K \cap \gamma K}\right)^{\prime}$.

\section{Examples of type-determining sets}

The assumption that $E$ is a centrally orthocomplete pseudoeffect algebra remains in force.

Recall that an atom in a pseudoeffect algebra $E$ is a nonzero element $a \in E$ such that if $x \leq a$ then either $x=0$ or $x=a$. A pseudoeffect algebra $E$ is atomic if and only if for every $e \in E$ there is an atom $a \leq e$. Let $A$ (which may be empty) denote the set of all atoms of $E$. 
LEMMA 6.1. If $a \in A$ is an atom in $E$, then $\gamma a$ is an atom in $\Gamma(E)$. Consequently, if $E$ is atomic, then $\Gamma(E)$ is atomic.

PROOF. Let $a \in A$ and $c \in \Gamma(E), c \leq \gamma a$. Then $c=\gamma(c \wedge a)$, so that $c=0$ if $c \wedge a=$ 0 , or $c=\gamma a$ if $c \wedge a=a$. If $E$ is atomic, then for every $c \in \Gamma(E) \subseteq E$ there is $a \in A$ with $a \leq c$, which yields $\gamma a \leq c$.

We say that an element $p \in E$, or equivalently, that $E[0, p]$ is atom-free if and only if $A \cap E[0, p]=\emptyset$.

LEMMA 6.2. [A] is the strongly type-determining subset of E generated by A.

Proof. If $A=\emptyset$, then $A^{\downarrow}=\emptyset$, otherwise $A^{\downarrow}=A \cup\{0\}$. In both cases, $\left[A^{\downarrow}\right]=[A]$, and the result follows from Theorem 5.2(ii).

An element of the strongly type-determining set $[A]$ is called a polyatom. The following theorem for centrally orthocomplete pseudoeffect algebras is analogous to [13, Theorem 4.7] for centrally orthocomplete effect algebras, and it enables us to decompose $E$ into atomic and atom-free parts.

THEOREM 6.3. The following properties hold.

(i) The set $A^{\prime}=[A]^{\prime}$ is strongly type-determining and consists of all atom-free elements of $E$.

(ii) The set $A^{\prime \prime}=[A]^{\prime \prime}$ is strongly type-determining and its nonzero part consists of elements $p \in E$ such that $E[0, p]$ is atomic.

(iii) $c_{A^{\prime} \cap \gamma\left(A^{\prime}\right)}=c_{[A]}^{\prime}$ is atom-free.

(iv) $A \subseteq[A] \subseteq E\left[0, c_{[A]}\right]$.

(v) If $p \in E$, then $p$ is atom-free if and only if $[A] \cap E[0, p]=\{0\}$.

(vi) $[A \cap \Gamma(E)]=[A] \cap \Gamma(E)$.

Proof. By Theorem 5.2(iii), $A^{\prime}$ and $A^{\prime \prime}$ are strongly type-determining subsets of $E$. Since $p \in A^{\prime}$ if and only if $p \wedge a=0$ for all atoms $a \in A, A^{\prime}$ is the set of all atomfree elements. Let $p \in A^{\prime \prime}$, then $q \wedge a=0$ for all $a \in A$ implies $q \wedge p=0$, hence if $p \wedge a=0$ for all $a \in A$, then $p=0$. Therefore if $0 \neq p \in A^{\prime \prime}$ then there is an atom $a \in A$ with $a \leq p$. This proves (i) and (ii). Part (iii) follows from (i) and Lemma 5.8.

(iv) If $a$ is an atom, then $a=\left(a \wedge c_{[A]}\right)+\left(a \wedge c_{[A]}^{\prime}\right)$, where $a \wedge c_{[A]}^{\prime}=0$ by part (iii). It follows that $a \leq c_{[A]}$. Therefore, $A \subseteq E\left[0, c_{[A]}\right]$, and since $E\left[0, c_{[A]}\right]$ is strongly type-determining, $[A] \subseteq E\left[0, c_{[A]}\right]$.

(v) Every atom is a nonzero polyatom, and a polyatom is nonzero if and only if it dominates an atom, hence $A \cap E[0, p]=\emptyset$ if and only if $[A] \cap E[0, p]=\{0\}$.

(vi) Since $[A]$ is a type-determining subset of $E$, so is $[A] \cap \gamma[A]=$ $[A] \cap \Gamma(E)$. Thus, as $A \cap \Gamma(E) \subseteq[A] \cap \Gamma(E)$, we have $[A \cap \Gamma(E)] \subseteq[A] \cap \Gamma(E)$. Let $h \in[A] \cap \Gamma(E)$. There is a $\Gamma$-orthogonal sequence $\left(a_{i}\right)_{i \in I}$ of atoms with $h=\sum_{i \in I} a_{i}=\bigvee_{i \in I} a_{i}$, since $h \in[A]$. Then the $\gamma a_{i}$, where $i \in I$, are pairwise orthogonal elements in $\Gamma(E)$, and since $h \in \Gamma(E), h=\gamma h=\bigvee_{i \in I} \gamma a_{i}=\sum_{i \in I} \gamma a_{i}$. 
It follows that $\sum_{i \in I} a_{i}=\sum_{i \in I} \gamma a_{i}$, and from $a_{i} \leq \gamma a_{i}$, for all $i \in I$, we deduce that $a_{i}=\gamma a_{i} \in \Gamma(E)$, and therefore $h \in[A \cap \Gamma(E)]$.

The notions of boolean and subcentral elements and monads were introduced in [11], and they also make sense in the setting of pseudoeffect algebras.

Definition 6.4. An element $b \in E$ is boolean if and only if $E[0, b]$ is a boolean algebra, that is, $E[0, b]=\Gamma(E[0, b])$.

By Lemma 3.12, for every $p \in E$ and $c \in \Gamma(E)$, the element $p \wedge c$ is central in $E[0, p]$. The next definition concerns those elements for which the converse also holds.

Definition 6.5. An element $p \in E$ is subcentral if and only if, for all $d \in \Gamma(E[0, p])$, $d=p \wedge c$ for some $c \in \Gamma(E)$.

Clearly, every central element is subcentral, and every atom is subcentral.

Definition 6.6. An element $h \in E$ is a monad if and only if, for every $e \in E[0, h]$, $e=h \wedge \gamma e$.

Notice that every atom is a monad. Similarly as in [13, Theorem 3.9], we obtain the following characterization of monads.

THEOREM 6.7. Let $h \in E$. Then the following properties are equivalent.

(i) $\quad h$ is a monad.

(ii) $h$ is both subcentral and boolean.

(iii) For all $e \in E[0, h], \gamma e=\gamma h$ only if $e=h$.

(iv) For all $e \in E[0, h], e^{\sim_{h}}, e^{-h} \leq(\gamma e)^{\prime}$.

(v) For all e, $f \in E[0, h], e+h f$ exists $\Leftrightarrow \gamma e \wedge \gamma f=0$.

Proof. We show first that (i) implies (ii). Let $h$ be a monad. Since $\Gamma(E[0, h]) \subseteq$ $E[0, h]$, if $d \in \Gamma(E[0, h])$, then $d=h \wedge \gamma d$, which shows that $h$ is subcentral. Since $e \in E[0, h]$ implies $e=h \wedge \gamma e$, and $\gamma e \in \Gamma(E)$, by Lemma 3.12, $e$ is central in $E[0, h]$, hence $e[0, h]=\Gamma(E[0, h])$, so $h$ is boolean.

Next, we show that (ii) implies (i). Since $h$ is subcentral, every $d \in \Gamma(E[0, h]$ is of the form $d=h \wedge c$ for some $c \in \Gamma(E)$. Then $d \leq c$ implies $\gamma d \leq c$, and consequently we see that $d=d \wedge \gamma d=h \wedge c \wedge \gamma d=h \wedge \gamma d$. As $h$ is also boolean, $\Gamma(e[0, h])=E[0, h]$, whence $d=h \wedge \gamma d$ holds for all $d \in E[0, h]$.

To show that (i) implies (iii), assume that $\gamma e=\gamma h, e \leq h$. Then $e=h \wedge \gamma e=$ $h \wedge \gamma h=h$.

Our fourth step is to show that (iii) implies (iv). Assume (iv), let $e \in E[0, h]$ and put $f:=e+\left(h \wedge(\gamma e)^{\prime}\right)$. As $e \leq \gamma e, h \wedge(\gamma e)^{\prime} \leq(\gamma e)^{\prime}$, and $\gamma e \in \Gamma(E)$, it follows that $f=e \vee\left(h \wedge(\gamma e)^{\prime}\right) \in E[0, h]$. Since $\gamma e \leq \gamma h$,

$$
\gamma f=\gamma e \vee \gamma\left(h \wedge(\gamma e)^{\prime}\right)=\gamma e \vee\left(\gamma h \wedge(\gamma e)^{\prime}\right)=(\gamma h \wedge \gamma e) \vee\left(\gamma h \wedge(\gamma e)^{\prime}\right)=\gamma h \text {, }
$$


so by (iii), $e+\left(h \wedge(\gamma e)^{\prime}\right)=f=h=e+e / h$ so $h \wedge(\gamma e)^{\prime}=e / h=e^{\sim_{h}} \leq(\gamma e)^{\prime}$. We can also write $f=\left(h \wedge(\gamma e)^{\prime}\right)+e=h=h \backslash e+e$, which yields the desired result: $h \wedge(\gamma e)^{\prime}=h \backslash e=e^{-h} \leq(\gamma e)^{\prime}$.

Now, we show that (iv) implies (v). Let $e, f \in E[0, h]$, and assume that $e+{ }_{h} f$ exists. Then $f \leq e^{-h} \leq(\gamma e)^{\prime}$, the last inequality following from (iv). Now $f \leq(\gamma e)^{\prime}$ implies $\gamma f \leq(\gamma e)^{\prime}$, which entails $(\mathrm{v})$.

Finally, we show that (v) implies (i). Let $e \in E[0, h]$, then $h=e+e^{\sim_{h}}=e+$ $(e / h)$, and by $(\mathrm{v}), \gamma(e / h) \leq(\gamma e)^{\prime}$. We also have $h=h \wedge \gamma e+h \wedge(\gamma e)^{\prime}$, and from $e \leq h \wedge \gamma e$ and $e / h \leq h \wedge(\gamma e)^{\prime}$ we deduce that $e=h \wedge \gamma e$, whence $h$ is a monad.

Let $S$ denote the set of all subcentral elements of $E, B$ the set of all boolean elements of $E$, and $H$ the set of all monads in $E$. As in [11], it can be shown that $S$ is a type-determining set with $[A] \subseteq S, B$ is a strongly type-determining set with $[A] \subseteq B$, and $H=S \cap B$ is a strongly type-determining set with $[A] \subseteq H$.

The following definition is an analogue of [13, Definition 4.2].

Definition 6.8. A nonempty class $\mathcal{K}$ of pseudoeffect algebras is called a type-class if and only if the following conditions are satisfied.

(i) $\mathcal{K}$ is closed under passage to direct summands, that is, if $H \in \mathcal{K}$ and $h \in \Gamma(H)$, then $H[0, h] \in \mathcal{K}$.

(ii) $\mathcal{K}$ is closed under the formation of arbitrary direct products.

(iii) If $E_{1}$ and $E_{2}$ are isomorphic pseudoeffect algebras and $E_{1} \in \mathcal{K}$, then $E_{2} \in \mathcal{K}$.

If $\mathcal{K}$ satisfies (ii), (iii); and

(i) $^{\prime} \quad H \in \mathcal{K}, h \in H$ implies that $H[0, h] \in \mathcal{K}$,

then $\mathcal{K}$ is called a strong type-class.

We omit the proof of the next theorem as it is analogous to that of [13, Theorem 4.4].

TheOREM 6.9. Let $\mathcal{K}$ be a type-class and define $K:=\{k \in E: E[0, k] \in \mathcal{K}\}$. Then $K$ is a type-determining subset of $E$. If $\mathcal{K}$ is a strong type-class, the $K$ is strongly type-determining.

EXAMPLE 6.10. The class of effect algebras and the following subclasses of effect algebras are strong type-classes: all boolean effect algebras, all orthomodular lattices, all complete orthomodular lattices, all orthoalgebras, all lattice effect algebras, and all atomic effect algebras. Similarly, all lattice-ordered pseudoeffect algebras and all atomic pseudoeffect algebras are strong type-classes.

According to [5], the pseudoeffect algebra $E$ is monotone $\sigma$-complete if and only if any ascending sequence $x_{1} \leq x_{2} \leq \cdots$ in $E$ has a supremum $\bigvee_{i=1}^{\infty} x_{i}$ in $E$; further, $E$ is $\sigma$-complete if and only if it is a $\sigma$-complete lattice; moreover, $E$ satisfies the countable Riesz interpolation property ( $\sigma$-RIP) if and only if, for countable sequences $\left\{x_{1}, x_{2}, \ldots,\right\}$ and $\left\{y_{1}, y_{2}, \ldots,\right\}$ of elements of $E$ such that $x_{i} \leq y_{j}$ for all $i, j$, there exists an element $z \in E$ such that $x_{i} \leq z \leq y_{j}$ for all $i, j$; and finally, $E$ is archimedean 
if and only if the only $x \in E$ such that $n x:=x+\cdots+x$ is defined in $E$ for any integer $n \geq 1$ is $x=0$.

One can easily deduce that monotone $\sigma$-complete pseudoeffect algebras, $\sigma$-complete pseudoeffect algebras, pseudoeffect algebras with the countable Riesz interpolation property, and archimedean pseudoeffect algebras are all strong typeclasses.

In [7], the following properties of pseudoeffect algebras were introduced.

Definition 6.11. Let $(E ;+, 0,1)$ be a pseudoeffect algebra.

(i) $E$ has the Riesz interpolation property (RIP) if and only if, for any $a_{1}, a_{2}, b_{1}, b_{2} \in E$ such that $a_{1}, a_{2} \leq b_{1}, b_{2}$, there is $c \in E$ such that $a_{1}, a_{2} \leq$ $c \leq b_{1}, b_{2}$.

(ii) $E$ has the weak Riesz decomposition property $\left(\mathrm{RDP}_{0}\right)$ if and only if, for any $a, b_{1}, b_{2} \in E$ such that $a \leq b_{1}+b_{2}$, there are $d_{1}, d_{2} \in E$ such that $d_{1} \leq b_{1}, d_{2} \leq$ $b_{2}$ and $a=d_{1}+d_{2}$.

(iii) $E$ has the Riesz decomposition property (RDP) if and only if, for any $a_{1}, a_{2}, b_{1}, b_{2} \in E$ such that $a_{1}+a_{2}=b_{1}+b_{2}$, there are $d_{1}, d_{2}, d_{3}, d_{4} \in E$ such that $d_{1}+d_{2}=a_{1}, d_{3}+d_{4}=a_{2}, d_{1}+d_{3}=b_{1}$, and $d_{2}+d_{4}=b_{2}$.

(iv) $E$ has the commutational Riesz decomposition property $\left(\mathrm{RDP}_{1}\right)$ if and only if, for any $a_{1}, a_{2}, b_{1}, b_{2} \in E$ such that $a_{1}+a_{2}=b_{1}+b_{2}$, there are $d_{1}, d_{2}, d_{3}, d_{4} \in$ $E$ such that $d_{1}+d_{2}=a_{1}, d_{3}+d_{4}=a_{2}, d_{1}+d_{3}=b_{1}, d_{2}+d_{4}=b_{2}$ and $x \leq$ $d_{2}, y \leq d_{3}$ imply $x+y=y+x$.

(v) $E$ has the strong Riesz decomposition property $\left(\mathrm{RDP}_{2}\right)$ if and only if, for any $a_{1}, a_{2}, b_{1}, b_{2} \in E$ such that $a_{1}+a_{2}=b_{1}+b_{2}$, there are $d_{1}, d_{2}, d_{3}, d_{4} \in E$ such that $d_{1}+d_{2}=a_{1}, d_{3}+d_{4}=a_{2}, d_{1}+d_{3}=b_{1}, d_{2}+d_{4}=b_{2}$ and $d_{2} \wedge d_{3}=0$.

In [7, Proposition 3.3], the following implications were proved:

$$
\left(\mathrm{RDP}_{2}\right) \Rightarrow\left(\mathrm{RDP}_{1}\right) \Rightarrow(\mathrm{RDP}) \Rightarrow\left(\mathrm{RDP}_{0}\right) \Rightarrow(\mathrm{RIP})
$$

In general, the converse of each of these implications fails. If $E$ is commutative (that is, an effect algebra), then $\left(\mathrm{RDP}_{1}\right),(\mathrm{RDP})$ and $\left(\mathrm{RDP}_{0}\right)$ are equivalent.

Since for any $k \in E$, if $a+b$ exists in $E[0, k]$ then $a+b$ exists in $E$, and the operations in direct products are defined pointwise, it is easy to deduce that pseudoeffect algebras with any of the properties from Definition 6.11 are strong typeclasses.

In [28], the following class of pseudoeffect algebras was introduced. An effect algebra $E$ is weak-commutative if, for any $a, b \in E, a+b$ exists if and only if $b+a$ exists. It was proved in [28] that in a weak-commutative pseudoeffect algebra, $a^{\sim}=$ $a^{-}$for every $a \in E$. On the other hand, if $a^{\sim}=a^{-}$, then $b \leq a^{\sim}$ if and only if $b \leq a^{-}$, whence $a+b$ exists if and only if $b+a$ exists for all $a, b \in E$. A weak-commutative pseudoeffect algebra becomes an effect algebra if and only if $a+b=b+a$ whenever one side of the equality exists. It was shown in [28] that effect algebras are a proper subclass of weak-commutative pseudoeffect algebras. 
THEOREM 6.12. The class of weak-commutative pseudoeffect algebras is a type-class which is not a strong type-class.

ProOf. Let $c \in \Gamma(E), a, b \in E[0, c]$. Then $a+b$ exists in $E[0, c]$ if and only if $a+b$ exists in $E$, so $b+a$ exists in $E$, whence $b+a$ exists in $E[0, c]$. Verification of the remaining properties of a type-class is straightforward.

Suppose that the class in question is a strong type-class. Then for every $d \in E$, $E[0, d]$ would be weak-commutative; hence if $a, b \leq d$ and $a+b \leq d$, then $b+a \leq d$. Putting $d=a+b$ yields $b+a \leq a+b$, and putting $d=b+a$ yields $a+b \leq b+a$.

In what follows we assume that $K$ and $F$ are type-determining subsets of the centrally orthocomplete pseudoeffect algebra $E$ and that $K \subseteq F$. As in Theorem 5.9, we decompose $E$ as

$$
E=E\left[0, c_{1}\right] \times E\left[0, c_{2}\right] \times E\left[0, c_{3}\right]
$$

and also as

$$
E=E\left[0, d_{1}\right] \times E\left[0, d_{2}\right] \times E\left[0, d_{3}\right]
$$

where $c_{1}=c_{K \cap \gamma K}$ and $d_{1}=c_{F \cap \gamma F}$ are of types $K$ and $F ; c_{2}=c_{K} \wedge c_{K \cap \gamma K}^{\prime}$ and $d_{2}=c_{F} \wedge c_{F \cap \gamma F}^{\prime}$ are locally types $K$ and $F$, but properly non- $K$ and properly non- $F$; and $c_{3}=c_{K}^{\prime}$ and $d_{3}=c_{F}^{\prime}$ are purely non- $K$ and purely non- $F$, respectively.

As $K \subseteq F$, it is clear that, type- $K$ implies type- $F$; locally type- $K$ implies locally type- $F$; purely non- $F$ implies purely non- $K$; and properly non- $F$ implies properly non- $K$.

The following theorem is an analogue of [13, Theorem 6.6] proved for effect algebras; since its proof in the pseudoeffect algebra setting follows the same ideas, we omit it.

THEOREM 6.13. There exists a direct sum decomposition

$$
E=E\left[0, c_{11}\right] \times E\left[0, c_{21}\right] \times E\left[0, c_{22}\right] \times E\left[0, c_{31}\right] \times E\left[0, c_{32}\right] \times E\left[0, c_{33}\right]
$$

where $c_{11}$ is type- $K$ (hence type- $F$ ); $c_{21}$ is type- $F$, locally type- $K$, but properly non- $K$; $c_{22}$ is locally type- $K$ (hence, locally type- $F$ ), but properly non- $F$ (hence, properly non-K); $c_{31}$ is type- $F$ and purely non- $K ; c_{32}$ is locally type- $F$ but properly non- $F$, and purely non-K; and $c_{33}$ is purely non- $F$ (hence, purely non-K). Moreover, such $a$ decomposition is unique, with $c_{i j}=c_{i} \wedge d_{j}$ for $i, j=1,2,3$, where $c_{11}=c_{1}, c_{33}=d_{3}$ and $c_{12}=c_{13}=c_{23}=0$.

In analogy with the classical decomposition of von Neumann algebras into types I, II, and III, we introduce the following definition (see also [13, Definition 6.3]).

DeFinition 6.14. For the type-determining sets $K$ and $F$ with $K \subseteq F$, the centrally orthocomplete pseudoeffect algebra $E$ is type $I$ if and only if it is locally type- $K$; type II if and only if it is locally type- $F$, but purely non- $K$; and type III if and only if 
it is purely non- $F$. It is type $\mathrm{I}_{F}$ or type $\mathrm{II}_{F}$ if and only if it is type $\mathrm{I}$ or type II and also type- $F$. It is type $\mathrm{I}_{\bar{F}}$ or type $\mathrm{II}_{\bar{F}}$ if and only if it is of type I or type II and also properly non- $F$.

The following theorem is the I/II/III-decomposition theorem for centrally orthocomplete pseudoeffect algebras.

Theorem 6.15. E decomposes as $E=E\left[0, c_{I}\right] \times E\left[0, c_{I I}\right] \times E\left[0, c_{I I I}\right]$, where $c_{I}$, $c_{I I}$ and $c_{I I I}$ are central elements of types I, II, and III; such a decomposition is unique, and $c_{I}=c_{K}, c_{I I}=c_{F} \wedge c_{K}^{\prime}, c_{I I I}=c_{F}^{\prime}$.

Moreover, there are further decompositions $E\left[0, c_{I}\right]=E\left[0, c_{I F}\right] \times E\left[0, c_{I \bar{F}}\right]$ and $E\left[0, c_{I I}\right]=E\left[0, c_{I I F}\right] \times E\left[0, c_{I I \bar{F}}\right]$, where $c_{I F}, c_{I \bar{F}}, c_{I I F}, c_{I I \bar{F}}$ are central elements of types $I_{F}, I_{\bar{F}}, I I_{F}, I I_{\bar{F}}$; these decompositions are also unique.

We obtain these decompositions if in Theorem 6.13 we put $c_{I}:=c_{11}+c_{21}+c_{22}$, $c_{I I}:=c_{31}+c_{32}$ and $c_{I I I}=c_{33} ;$ then $c_{I F}:=c_{11}+c_{21}, \quad c_{I \bar{F}}:=c_{22}, \quad c_{I I F}:=c_{31}$, $c_{I I \bar{F}}:=c_{32}$. Notice that, beyond the traditional I/II/III decomposition, the type $\mathrm{I}_{F}$ summand decomposes as $E\left[0, c_{I F}\right]=E\left[0, c_{11}\right] \times E\left[0, c_{21}\right]$, where $c_{11}$ is type- $K$ (hence type- $F$ ) and $c_{21}$ is type- $F$ and locally type- $K$, but properly non- $K$.

EXAMPLE 6.16. Taking $K:=[A]$, the set of all polyatoms, and $F:=H$, the set of all monads of $E$, in Theorem 6.15, we deduce that $[A] \subseteq H$, and $E$ decomposes as $E=E\left[0, r_{1}\right] \times E\left[0, r_{2}\right] \times E\left[0, r_{3}\right]$ where every nonzero direct summand of $E\left[0, r_{1}\right]$ contains an atom; $E\left[0, r_{2}\right]$ is atom-free, but every nonzero direct summand of $E\left[0, r_{2}\right]$ contains a nonzero monad; and $E\left[0, r_{3}\right]$ contains no nonzero monad. This decomposition is unique. Indeed, $r_{1}=c_{[A]}$ is locally type- $[A], r_{2}=c_{H} \wedge c_{[A]}^{\prime}$ is locally type- $H$ and purely non- $[A]$, and $r_{3}=c_{H}^{\prime}$ is purely non- $H$ (see Theorem 6.15).

EXAMPLE 6.17. Take $K=: E A$, the subset of all elements $e \in E$ such that $E[0, e]$ is a commutative pseudoeffect algebra (that is, an effect algebra), and $F=: W$, the set of all elements $d \in E$ such that $E[0, d]$ is weak-commutative. Then $E A \subseteq W$, and we obtain the decomposition $E=E\left[0, v_{1}\right] \times E\left[0, v_{2}\right] \times E\left[0, v_{3}\right]$. The summand $E\left[0, v_{1}\right]$ is locally commutative in the sense that $v_{1}=\gamma e=c_{E A}$; the summand $E\left[0, v_{2}\right]$ is locally weak-commutative, but purely noncommutative, that is, $v_{2}=$ $c_{W} \wedge c_{E A}^{\prime}$; and $E\left[0, v_{3}\right]$ is purely nonweak-commutative, that is, $v_{3}=c_{W}^{\prime}$. We recall that then every direct subsummand of $E\left[0, v_{1}\right]$ contains an element $e \in E A$; every direct subsummand of $E\left[0, v_{2}\right]$ contains an element $d \in W$, but $E\left[0, v_{2}\right] \cap E A=\{0\}$; and $E\left[0, v_{3}\right]$ contains no element of $W$.

The summands $E\left[0, v_{1}\right]$ and $E\left[0, v_{2}\right]$ decompose further into weak-commutative and properly nonweak-commutative parts; and the weak-commutative part of $E\left[0, v_{1}\right]$ admits a further decomposition into a commutative part and a locally commutative but properly noncommutative part.

Let $R 2$ denote the strongly type-determining set of all elements $e \in E$ such that $E[0, e]$ satisfies $\left(\mathrm{RDP}_{2}\right)$ and $L$ denote the set of all elements $e \in E$ such that $E[0, e]$ is a lattice. 
EXAMPLE 6.18. There exists a decomposition

$$
E=E\left[0, c_{11}\right] \times E\left[0, c_{21}\right] \times E\left[0, c_{22}\right] \times E\left[0, c_{31}\right] \times E\left[0, c_{32}\right] \times E\left[0, c_{33}\right],
$$

where $E\left[0, c_{11}\right]$ satisfies $\left(\mathrm{RDP}_{2}\right)$, hence is a lattice; $E\left[0, c_{21}\right]$ is a lattice, and every direct subsummand contains an element from $R 2$, but no direct subsummand satisfies $\left(\mathrm{RDP}_{2}\right) ; E\left[0, c_{22}\right]$ contains no lattice ordered direct subsummand (hence no subsummand satisfying $\left.\left(\mathrm{RDP}_{2}\right)\right)$, but every direct subsummand contains an element from $R 2$ (hence from $L$ ); $E\left[0, c_{31}\right]$ is a lattice and contains no element from $R 2$; $E\left[0, c_{32}\right]$ contains no lattice ordered direct subsummand, and no element from $R 2$, but every direct subsummand contains an element from $L$; and $E\left[0, c_{33}\right]$ contains no element from $L$ (hence no element from $R 2$ ). Moreover, such a decomposition is unique.

Indeed, such a decomposition is obtained from decompositions corresponding to strongly type-determining sets $R 2$ and $L$ as in Theorem 6.15, taking into account that $R 2 \subseteq L$ by [7, Proposition 3.3(ii)].

Notice that by [8], a pseudoeffect algebra that satisfies $\left(\mathrm{RDP}_{2}\right)$ is a pseudo-MV algebra (a noncommutative analogue of an MV algebra; see [14, 24]).

\section{References}

[1] L. Beran, Orthomodular Lattices, An Algebraic Approach, Mathematics and its Applications, 18 (D. Reidel, Dordrecht, 1985).

[2] J. C. Carrega, G. Chevalier and R. Mayet, 'Direct decompositions of orthomodular lattices', Algebra Universalis 27 (1990), 480-496.

[3] C. C. Chang, 'Algebraic analysis of many-valued logic', Trans. Amer. Math. Soc. 88 (1958), $467-490$.

[4] J. Dixmier, Les algèbres d'opérateurs dans l'espace hilbertien (Gauthier-Villars, Paris, 1957).

[5] A. Dvurečenskij, 'Central elements and Cantor-Bernstein's theorem for pseudo-effect algebras', J. Aust. Math. Soc. 74 (2003), 121-143.

[6] A. Dvurečenskij and S. Pulmannová, New Trends in Quantum Structures (Kluwer, Dordrecht, 2000).

[7] A. Dvurečenskij and T. Vetterlein, 'Pseudoeffect algebras. I. Basic properties', Internat. J. Theoret. Phys. 40 (2001), 685-701.

[8] A. Dvurečenskij and T. Vetterlein, 'Pseudoeffect algebras. II. Group representations', Internat. J. Theoret. Phys. 40 (2001), 703-726.

[9] A. Dvurečenskij and T. Vetterlein, 'Non-commutative algebras and quantum structures', Internat. J. Theoret. Phys. 43(7/8) (2004), 1599-1612.

[10] D. J. Foulis and M. K. Bennett, 'Effect algebras and unsharp quantum logics', Found. Phys. 24(10) (1994), 1331-1352.

[11] D. J. Foulis and S. Pulmannová, 'Centrally orthocomplete effect algebras', Algebra Universalis, DOI 10.1007/S00012-010-0100-5.

[12] D. J. Foulis and S. Pulmannová, 'Hull mappings and dimension effect algebras', Math. Slovaca, to appear.

[13] D. J. Foulis and S. Pulmannová, 'Type decomposition of an effect algebra', Found. Phys. 40 (2010), 1543-1565.

[14] G. Georgescu and A. Iorgulescu, 'Pseudo-MV algebras: a non-commutative extension of MV algebras', in: Proc. Fourth Inter. Symp. on Econ. Inform., May 6-9, Bucharest (eds. I. Sumeurenau et al.) (INFOREC Printing House, Bucharest, 1999), pp. 961-968. 
[15] K. R. Goodearl and F. Wehrung, 'The complete dimension theory of partially ordered systems with equivalence and orthogonality', Mem. Amer. Math. Soc. 176(831) (2005).

[16] R. J. Greechie, D. J. Foulis and S. Pulmannová, 'The center of an effect algebra', Order 12 (1995), 91-106.

[17] G. Kalmbach, Orthomodular Lattices (Academic Press, New York, 1983).

[18] G. Kalmbach, Measures and Hilbert Lattices (World Scientific, Singapore, 1986).

[19] I. Kaplansky, 'Projections in Banach algebras', Ann. of Math. (2) 53(2) (1951), 235-249.

[20] I. Kaplansky, Rings of Operators (W.A. Benjamin, New York and Amsterdam, 1968).

[21] L. H. Loomis, The Lattice Theoretic Background of the Dimension Theory of Operator Algebras, Memoirs of the American Mathematical Society, 18 (American Mathematical Society, Providence, RI, 1955).

[22] S. Maeda, 'Dimension functions on certain general lattices', J. Sci. Hiroshima Univ. A19 (1955), 211-237.

[23] J. von Neumann, 'On rings of operators', J. von Neumann Collected Works, Vol. III (Pergamon Press, Oxford, 1961).

[24] J. Rachůnek, 'A non-commutative generalization of MV algebras', Czechoslovak Math. J. 52 (2002), 255-273.

[25] A. Ramsay, 'Dimension theory in complete weakly modular orthocomplemented lattices', Trans. Amer. Math. Soc. 116 (1965), 9-31.

[26] R. Sikorski, Boolean Algebras, 2nd edn (Academic Press, New York and Springer, Berlin, 1964).

[27] D. M. Topping, Jordan Algebras of Self-adjoint Operators, Memoirs of the American Mathematical Society, 53 (American Mathematical Society, Providence, RI, 1965).

[28] Y. Xie and Y. Li, 'Central elements in pseudo-effect algebras', Math. Slovaca, to appear.

DAVID J. FOULIS, Department of Mathematics and Statistics, University of Massachusetts, Amherst, MA, USA

e-mail: foulis@math.umass.edu

and

Current Address: 1 Sutton Court, Amherst, MA 01002, USA

SYLVIA PULMANNOVÁ, Mathematical Institute, Slovak Academy of Sciences, Stefánikova 49, SK-814 73 Bratislava, Slovakia

e-mail: pulmann@mat.savba.sk

ELENA VINCEKOVÁ, Mathematical Institute, Slovak Academy of Sciences, Stefánikova 49, SK-814 73 Bratislava, Slovakia

e-mail: vincek@mat.savba.sk 